\title{
Asymmetries, Correlations and Fat Tails in Percolation Market Model
}

\author{
Iksoo Chang $^{1}$, Dietrich Stauffer ${ }^{1,2,3}$, and Ras B. Pandey ${ }^{2}$ \\ ${ }^{1}$ Department of Physics, Pusan National University, Busan 609-735, South Korea \\ 2 Department of Physics and Astronomy, University of Southern Mississippi, Hattiesburg \\ MS39406-5046, USA \\ ${ }^{3}$ Institute for Theoretical Physics, Cologne University, D-50923 Köln, Euroland
}

\begin{abstract}
Modifications of the Cont-Bouchaud percolation model for price fluctuations give an asymmetry for time-reversal, an asymmetry between high and low prices, volatility clustering, effective multifractality, correlations between volatility and traded volume, and a power law tail with exponent near 3 for the cumulative distribution function of price changes. Combining them together still gives the same power law. Using Ising-correlated percolation does not change these results. Different modifications give log-periodic oscillations before a crash, arising from nonlinear feedback between random fluctuations.
\end{abstract}

\section{Introduction}

Fluctuations for stockprices or other markets have statistical properties [1] which should be recovered by suitable microscopic models [2]: (i) There are only weak correlations between the price changes on successive trading days; (ii) there are strong correlations between the absolute values of the price changes on successive trading days ("volatility clustering"); (iii) the probability distribution function for the price changes (the return histogram) decays with a power law in the tails [3, 4], with exponent near 3; (iv) price fluctuations show sharp peaks and flat valleys, i.e. a high-low asymmetry [5]; (v) price fluctuations are not invariant against time reversal, i.e. they show a forward-backward asymmetry [6]; (vi) for long times (many weeks) a crossover to a more Gaussian return histogram is seen [4]; (vii) the $q$-th moments of the return histograms show multifractality, i.e. their time exponents are not a linear function of $q$.

The Cont-Bouchaud model [7] uses random percolation clusters as groups of traders buying or selling together and thus simulates human herding. In the simple version, at each iteration each cluster buys with probability $a$, sells with probability $a$, or sleeps with probability $1-2 a$. This activity thus measures the time between two iterations: small $a$ mean short times and large $a$ mean long times. Price changes (more precisely, 
changes in the logarithm of the price, where the prices are measured in units of some fundamental price) are proportional to the difference between supply and demand. By construction, on average one has as many buyers as sellers, and prices go up or down with equal probability and without correlations between consecutive steps, fulfilling (i). Increasing $a$ from low values to its maximum value $1 / 2$ changes the return histogram from power-law tails to a more Gaussian shape [8], fulfilling property (vi). Changing a proportionally to the last price change fulfills properties (ii) and (iv). Making the ratio of buying and selling probabilities different from unity and depending on the last known price and price change [9] fulfilled (v) and even gave log-periodic oscillations [10] after the crash of a price bubble [11]. Averaging over concentrations both near to and far away from the percolation threshold together with a square-root dependence of price changes on the difference between supply and demand gave a cumulative histogram of returns $r(=$ relative price change) with tail decaying asymptotically as $|r|^{-\mu}$ where $\mu=2(\tau+\sigma-1) \simeq 3$, fulfilling (iii). Thus all desired properties (i) to (vi) were fulfilled by one or the other modification; we are not aware of another microscopic model fulfilling all. (Perhaps the Levy-Solomon-Huang model can also give all these properties [12].)

The aim of the present work is to combine these modifications and to check which properties are then still valid in this combination model. We also check the influence of Ising correlations on the traders (occupied sites) on the lattice [13].

\section{Model}

An $L \times L$ triangular lattice (known to be in the same universality class as the more often studied square lattice) is occupied randomly (or later with Ising correlations) with probability $p$, which in our simulations does not exceed the percolation threshold $p_{c}=1 / 2$ at which an infinite cluster is formed. Clusters are sets of neighbouring occupied sites; right at $p=p_{c}$ the number $n_{s}$ of large clusters containing $s$ sites decays as $1 / s^{\tau}$ while near

$p_{c}$ it is $s^{-\tau} f\left[\left(p-p_{c}\right) s^{\sigma}\right]$ according to standard percolation theory [15] with $\tau \simeq 2, \sigma \simeq 0.4$ in two dimensions.

For market simulation, each occupied site is regarded as an agent (trader, investor), and clusters are companies of traders buying and selling together an amount proportional to the number $s$ of agents in the cluster. Each cluster is active with probability $2 a$ and inactive with probability $1-2 a$ during each time step independently; if it is active it buys with probability $p_{b}$ and sells with probability $p_{s}=1-p_{b}$, where $p_{b}=p_{s}=1 / 2$ only in the simple unbiased version [7]. Thus each cluster buys, sells, or sleeps with probabilities $2 a p_{b}, 2 a p_{s}, 1-2 a$, respectively. The total demand (supply) is $\sum_{s} n_{s} s$ with the sum running over all buying (selling) clusters and excluding the largest cluster. The price 
change $r$ at one time step is proportional to the difference of demand and supply, or to the square root [16] of that difference. We interpret the price change as a relative one, more precisely, this return

$$
r(t)=x(t)-x(t-1)
$$

is proportional to the change in the logarithm $x$ of the price (in units of an initial or fundamental price) and thus together with $x$ fluctuates about zero, in arbitrary units. In the simplest model we assume

$$
r=\sum_{b u y} n_{s} s-\sum_{\text {sell }} n_{s} s
$$

We take into account the agent psychology who believe that past trends will continue, but also their more reasonable wish to buy (sell) if $x$ is low (high). Thus if $r<0$ ( $r$ and $x$ are measured at the previous time step) we take at first

$$
p_{b}=0.5-5 \cdot 10^{-7} x+5 \cdot 10^{-4} r
$$

while for $r>0$ we take

$$
p_{b}=0.5-5 \cdot 10^{-7} x+5 \cdot 10^{-5} r
$$

The difference between these two equations is one order of magnitude for their last term and takes into account that agents are risk adverse and thus more impressed by a downturn than by an upturn of the market. With these parameters at $p=p_{c}$, a reasonable slight asymmetry between up and down for the prices was simulated [9].

To avoid the assumption that agents act only at the percolation threshold, we follow 177 and sum up over all $p$ between zero and the threshold, in units of one percent; and we take Zhang's square-root law [16]:

$$
r^{2}=\sum_{\text {buy }} n_{s} s-\sum_{\text {sell }} n_{s} s
$$

( $|r|$ is then rounded downward to an integer). With these assumptions but without the bias of eqs $(1,2)$ (i.e. with $\left.p_{b}=p_{s}=0.5\right)$ the probability $P(r)$ for a change $r$ decays as $r^{1-2 \tau-2 \sigma}=1 / r^{3.9}$ as desired [3], [1, for $r \gg 1$ (and $r$ much smaller than an upper limit given by cluster radius $=L$ ).

Assuming [18, in accordance with reality [19], the activity $a$ to increase with positive returns $r$,

$$
a(t+1)=a(t)+0.5 r(t) / L^{2}
$$




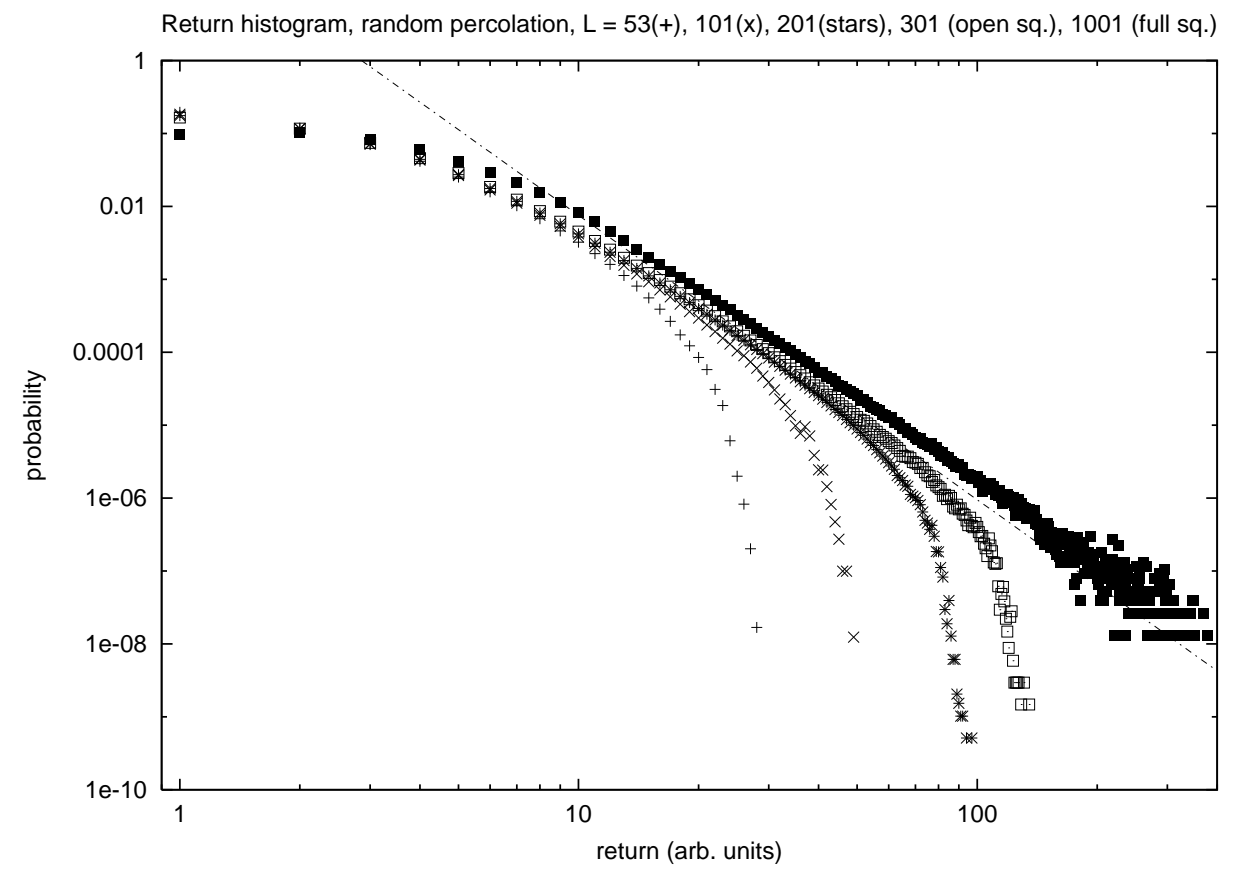

Figure 1: Probability $P(|r|)$ to have a return $|r|$ averaged over positive and negative values, for various $L$ as given in the headline. We simulated 1000 lattices for $L=53$ and 101, 20000 for 201, 6400 for 301, and 640 for 1001. For each lattice, 5000 time steps were made where each cluster decided to buy, sell or sleep.

(but always $1 / 10^{4}<a<1 / 2$ ) gives sharp peaks compared to flat valleys in $x(t)$ [5] and volatility clustering [18], at $p=p_{c}$ without the complications of eqs(1-3).

For Ising correlated agents [13, we no longer distribute agents randomly on a lattice but identify them with up spins of the standard Ising model at temperature $T$. Earlier [13], this temperature was varied between $T_{c}$ and infinity, leading always to $p=1 / 2$ for the fraction of occupied lattice sites. The results were similar to random occupation. Now, closer to [17] we take much smaller $p$ by increasing $T$ from $0.9 T_{c}$ to $1.01 T_{c}$ and thus $p$ from 0.06 to about 0.5, starting with all sites empty in Glauber kinetics. (Since the spontaneous magnetization $1 / 2-p$ at $T=T_{c}$ is about $1 / L^{1 / 8}$ and thus still quite large for realistic market sizes $L$, we took the upper limit at $1.01 T_{c}$ to push $p$ closer to $1 / 2$.)

Now we check if a combination of all these modification still gives reasonable results. 


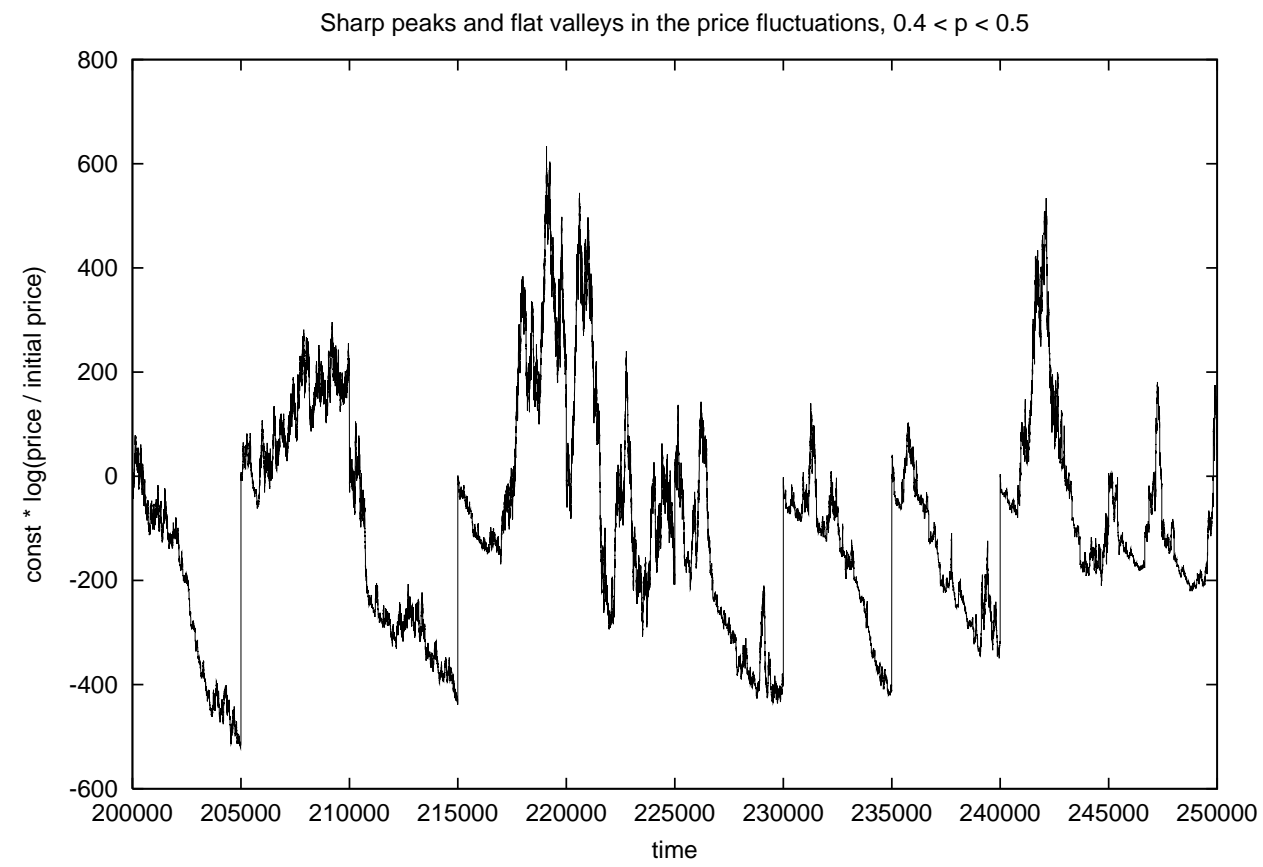

Figure 2: Last part of the price versus time curve for $L=301$. For each of the 50 concentrations $p$ up to $p_{c}=50$ percent, 5000 time steps were simulated for one lattice. The straight line has the theoretical slope 3.9.

\section{Results}

We combine modifications (1-4) and sum up over random occupation probabilities from one to 50 percent. Fig. 1 shows that for large enough markets the tail exponent is close to the desired 3.9. Fig.2 contrasts the resulting sharp peaks with the flatter valleys of $x(t)$. Fig.3 shows volatility clustering, i.e. large values of $|r|$ have a tendency to cause large $|r|$ thereafter. (If $r$ is not plotted logarithmically, the results are symmetric about zero.) The same figure shows that large $r$ are correlated with large activity $a$ in agreement with reality [20]. Unfortunatly, the asymmetry in time-reversal [9] is destroyed by assumptions (3) or (4); when we make the small prefactor of $r$ by which eqs $(1,2)$ differ ten times larger, some asymmetry is seen again.

The probability of a downward movement followed by an upward movement [16] is diminished by one to two percent compared to the three other choices, for 1000 lattices of $L=53$ and 101 . 


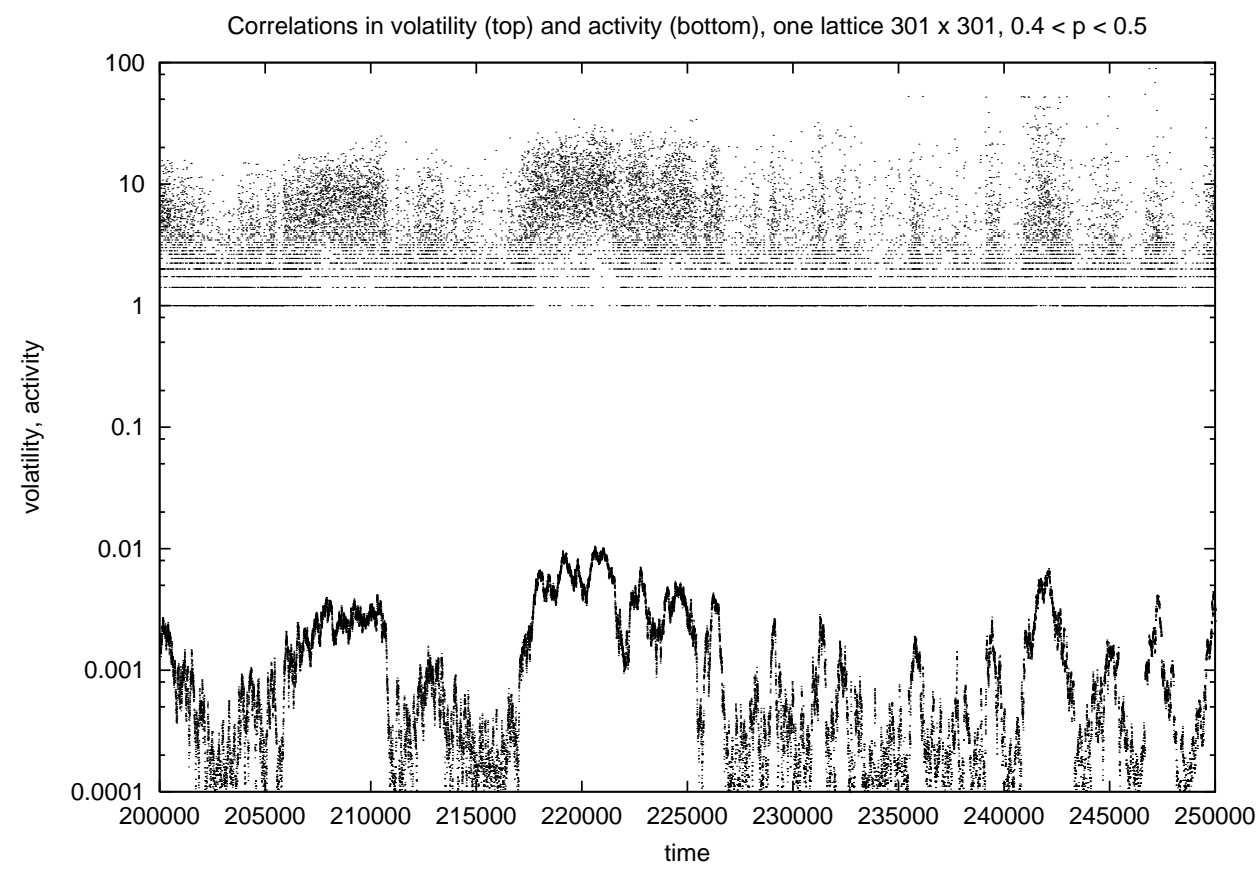

Figure 3: Same simulation as in Fig.2, but returns $r$ (top) and activity $a$ versus time. When the activity is large, the price changes are also large. Moreover, times with large $a$ and $r$ cluster together.

The crossover to a more Gaussian $P(r)$ for increasing activity $a$ is also no longer seen since now a large $a$ only means a large initial activity. Due to modification (4), $a$ decreases about linearly with time during the first half of the simulation (small $p$, small clusters and thus small $r$ ), and then fluctuates strongly near $1 / 10^{4} \ldots 1 / 10^{2}$ when market fluctuations become important. These low activities in the second half destroy the Gaussian behaviour.

For Ising-correlated agents the results are similar; Fig. 4 shows the fat tails.

Multifractality [21] here refers to the $q$-th moments $<|x(t+\tau)-x(t)|^{q}>_{t} \propto \tau^{\zeta}$ where for each $p$ separately we average over long times $t$. If $\zeta=\zeta(q)$ is linear in $q$ we have usual scaling; otherwise we have multifractality (multiaffinity, multiscaling, property (vii)). Fig.5 suggests multifractality in even better agreement with reality than for the unmodified Cont-Bouchad model [22]. In both cases the exponents are effective and might differ from the asymptotic behaviour [23].

In summary, properties (i) to (iv) are recovered, while the asymmetry (v) is partially and the crossover towards Gaussians is totally destroyed. 


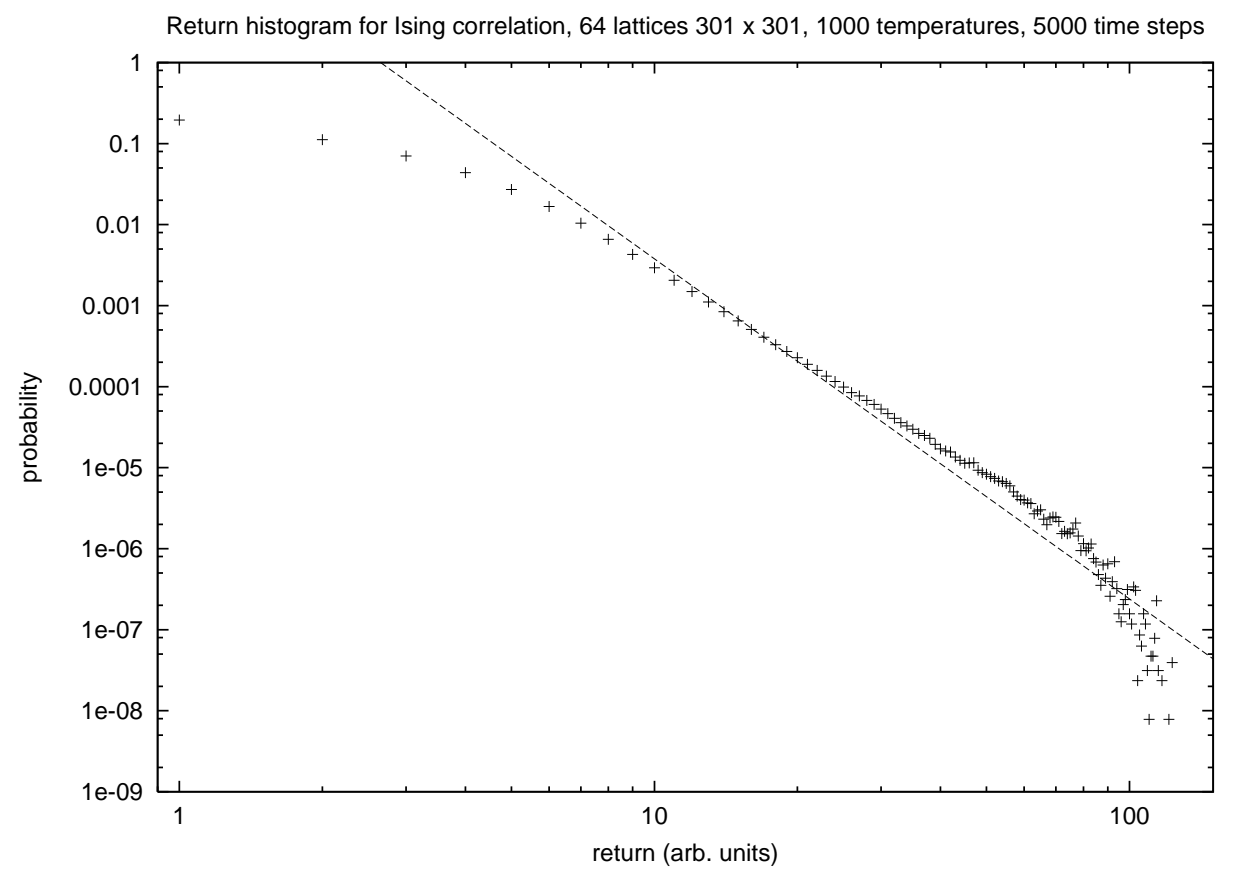

Figure 4: Similar to Fig.1, but for Ising correlation and 1000 temperatures for $L=301$. The straight line has the theoretical slope 4.2 .

\section{Log-periodic precursors of market crashes}

Before or after a stock market crash, one may observe log-periodic oscillations of the price. Major crashes are quite rare, perhaps several in a century for one given market. They may be outliers [24] not described by the above model for normal behaviour. Thus we use now a related but different model for log-periodicity.

One of the microscopic models to show them was our non-linear restoring and inertia force [11] : If prices go up (down), people have the tendency to buy (sell); this herding (inertia, hysteria,... ) enhances the trend and destabilizes the market. On the other hand, when prices are high, people have a tendency to sell, while they prefer to buy if prices are low; this restoring force stabilizes the market. When the restoring force was nonlinear, proportional to the fifth power of the deviation from the perceived fundamental price, and when the market started with a far too high price, then several oscillations were observed whose period increased exponentially with the order of the oscillation, until random noise 


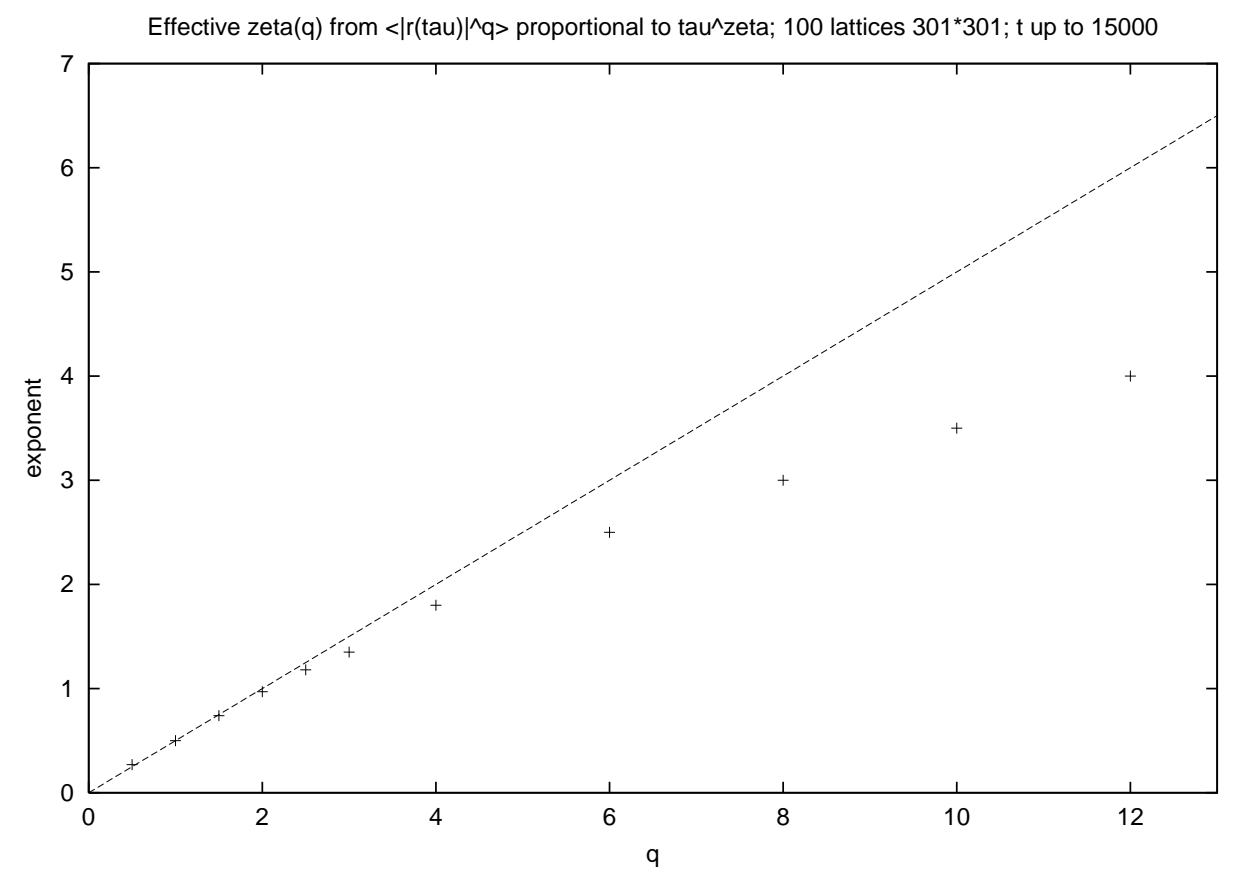

Figure 5: Effective exponents $\zeta$ versus $q$, from $10^{3}<\tau<3750$, for the moments $<|r(\tau)|^{q}>\propto \tau^{\zeta}$ with $r(\tau)=<x(t+\tau)-x(t)>_{t}$. The straight line $\zeta=q / 2$ would hold for random walks, while the downward curvature shows (effective) multifractality.

took over:

$$
\log (y)=x \propto \cos (\lambda \log t) * D
$$

where $y$ is the ratio of the actual price at time $t$ to the constant fundamental price, and $D$ is a damping factor, like $\log D \propto-t$. The actual price changes were produced by selling and buying clusters in a Cont-Bouchaud type model, where instead of geometrical clusters simply step sizes $s$ with a probability $n_{s}=N / s^{5 / 2}$ (Levy walks) were assumed.

A more phenomenological and simpler, but also more general approach [25] used the differential equation

$$
d^{2} x / d t^{2}=c_{m}(d x / d t)^{m}-c_{n} x^{n}
$$

with not only the restoring force $x^{n}$ but also the inertia $(d x / d t)^{m}$ being nonlinear. (If $m$ and $n$ are not odd integers, suitable signs and absolute values have to be used.) Now a small perturbation may grow until $x \rightarrow \pm \infty$ at some finite crash time $t_{c}$. We added a random noise \pm 0.002 to the RHS of this differential equation and then found results as in Fig.6, where three samples differing only in their random number sequences for 


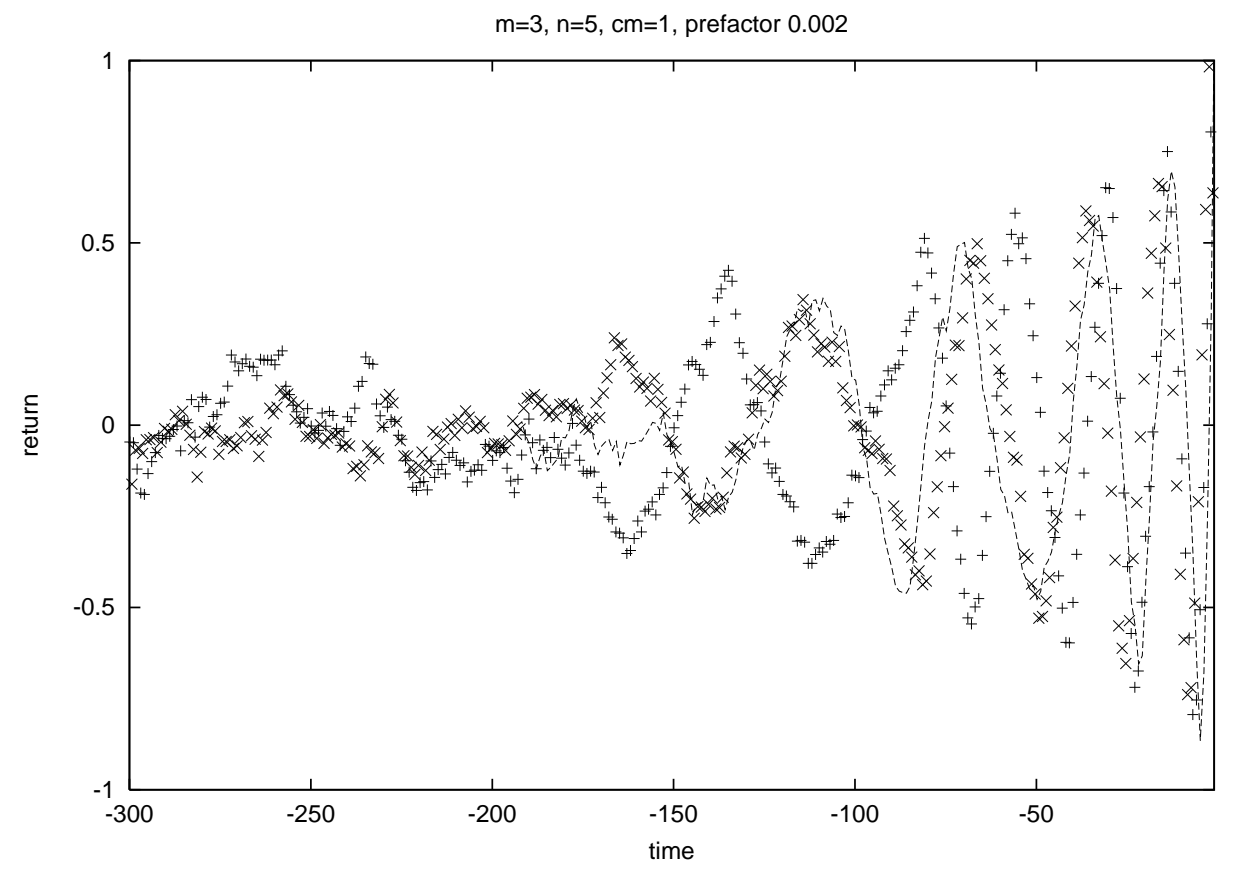

Figure 6: Three examples of random nonlinear behaviour, eq.(6) plus noise, leading to a crash; only the random numbers differ.

the noise are plotted versus $t-t_{c}$. We see that shortly before the singularity they show roughly the same maxima and minima, with the time between the extrema decreasing when $t$ approaches $t_{c}$. For earlier times the noise dominates, and the three curves differ significantly.

We apply these new ideas [25] to our old model [11] and take as probability $p_{b}$ to buy:

$$
p_{b}=0.5-c_{n} x^{n}+c_{m}(d x / d t)^{m}
$$

where the derivative in this discrete model means the price change from one iteration to the next. Thus each cluster sleeps with probability $1-2 a$, and if it does not sleep it buys with probability $p_{b}$ and sells with probability $1-p_{b}$, where $0<a<1 / 2$ is the activity. Initially, $x$ and $d x / d t$ are zero; prices and their changes are normalized by $N$. We then see for suitable parameters, like $a=1 / 4, m$ slightly above 1 and $c_{m}=0.5$ $\left(c_{n}=0.1\right.$ to 1 and $m=2$ to 5 seem less crucial), that after $10^{2}$ to $10^{3}$ iterations of random fluctuations, some oscillations emerge which become faster and faster, until we reach a crash at $t=t_{c}$ defined as $p_{b}=0$. Fig.7 shows average prices over 1000 samples, 


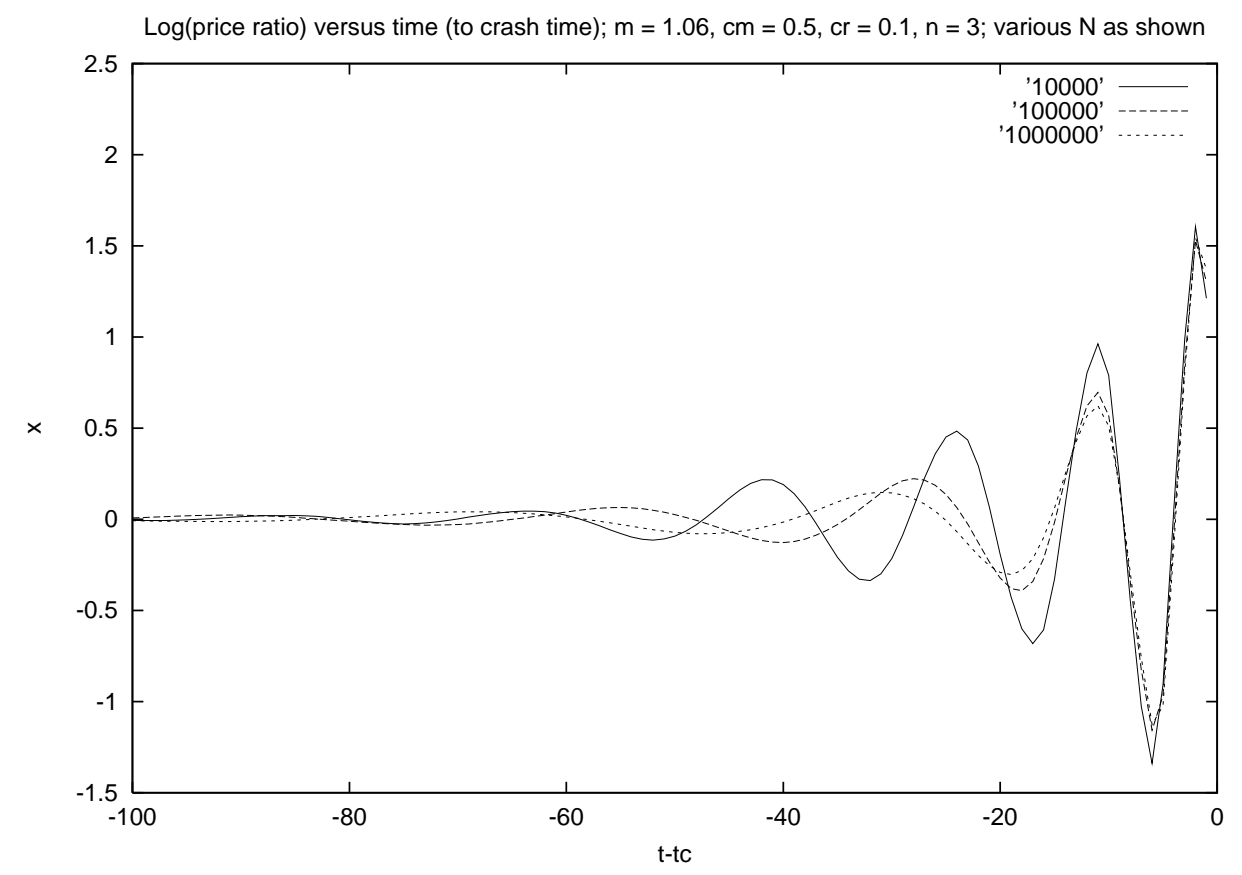

Figure 7: Average over thousand samples of the full model, for various market sizes as shown.

with market size $N=10^{4} \ldots 10^{6}$, where the random fluctuations mostly cancel each other while the oscillations shortly before the crash do not cancel. (The crash times are lognormally distributed.) Fig. 8 shows the resulting times where $x=0$; for large $N$ they indicate log-periodicity.

Qualitatively similar results are also observed with a Cont-Bouchaud type of cluster trading model, if the amount of stocks to buy is proportional to $x$, the solution of eq(6) with a small noise \pm 0.002 added to $x$.

\section{Conclusion}

Most of the desired properties are recovered, if we combine in the Cont-Bouchaud percolation model several of the modifications introduced in the past to get specific properties. Thus this modified model has become quite realistic in giving fat tails with proper exponent, multifractality, high-low asymmetry, volatility clustering and volatility-activity correlation. The crossover towards Gaussian behaviour needs some new ideas. In a dif- 


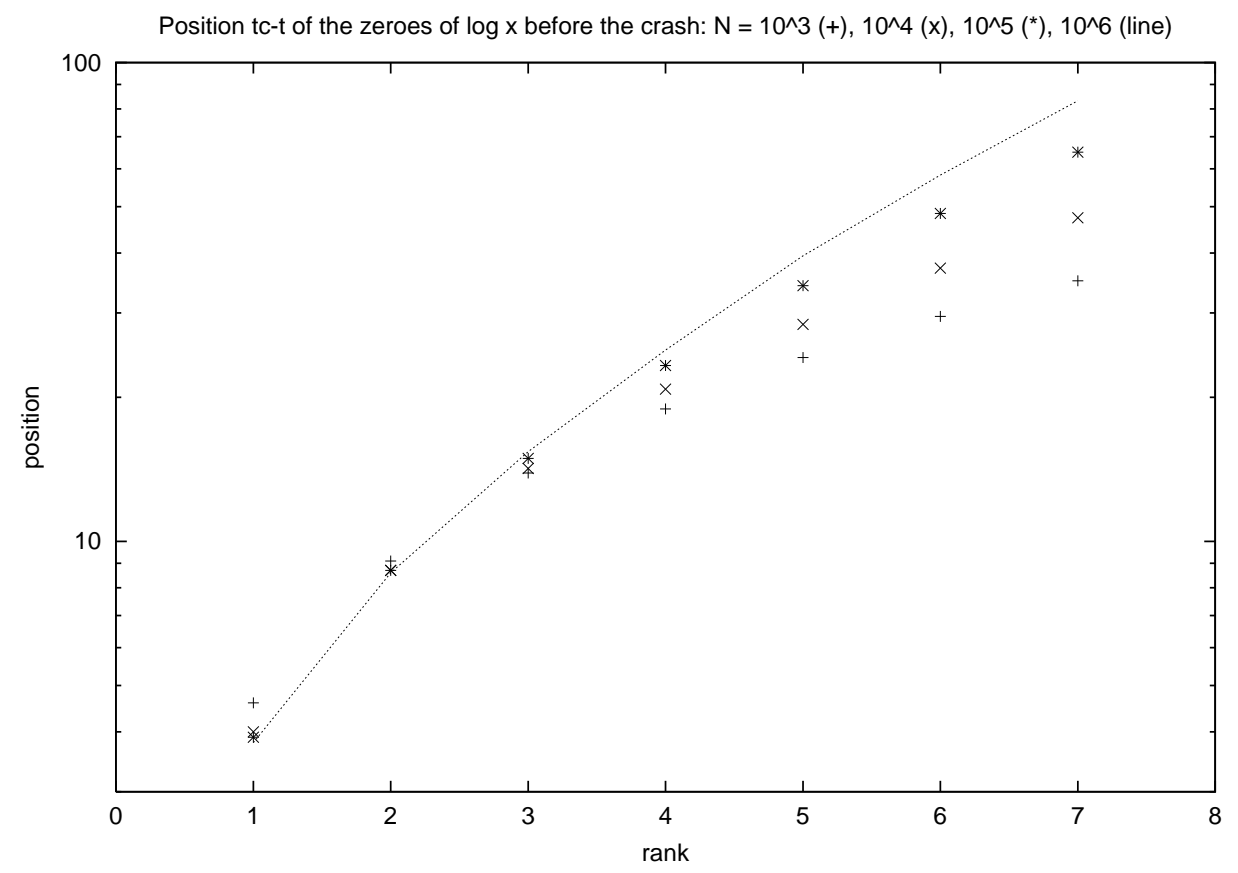

Figure 8: Position of zeroes in $x$ versus time shortly before the crash; perfect logperiodic oscillations would correspond to straight lines in this semilogarithmic plot and are achieved better for large markets.

ferent form it gives log-periodic oscillations before a crash.

Some of the simulations were made on the Cray-T3E of the Julich supercomputer center. DS thanks the two other authors for their hospitality, partly financed through KOSEF-DFG, during the time of this work.

\section{References}

[1] R. Cont, Quant. Finance 1, 223 (2001)

[2] M. Levy, H. Levy and S. Solomon, Microscopic Simulation of Financial Markets, Academic Press, New York 2000.

[3] T. Lux, Appl. Financial Economics 6, 463 (1996) and Empirical Economics 25, 641 (2000) 
[4] P. Gopikrishnan, V. Plerou, A.N. Amaral, M. Meyer and H.E. Stanley, Phys. Rev. E 60, 5305 (2000)

[5] B.M. Roehner and D. Sornette, Eur. Phys. J. B 4, 387 (1998)

[6] J.-F. Muzy, D. Sornette, J. Deloux and A. Arneodo, Quant. Finance 1, 131 (2001); A. Arneodo, J.F. Muzy, D. Sornette, Eur. Phys. J. B 2, 277 (1998)

[7] R. Cont and J.-P. Bouchaud, Macroeconomic Dynamics 4, 170 (2000). For a review of Monte Carlo simulations of this model see D. Stauffer, Adv. Complex Systems 4, 19 (2001).

[8] D. Stauffer and T.J.P. Penna, Physica A 256, 284 (1998); for a theory see L.Kullmann and J.Kertész, Int. J. Mod. Phys. C 12, No. 8 (2001)

[9] I. Chang and D. Stauffer, Physica A, in press (2001)

[10] D. Sornette and A. Johansen, Quant. Finance, in press

[11] R.B. Pandey and D. Stauffer, Int. J. Pure Appl. Finance 3, 279 (2000)

[12] Z.F. Huang and S. Solomon, Eur. J. Phys. 20, 601 (2001) with earlier literature generalized Lotka-Volterra models.

[13] L.R. da Silva and D. Stauffer, Physica A 294, 235 (2001); see also D. Chowdhury and D. Stauffer, Eur. J. Phys. B 8, 477 (1999); F. Castiglione, R.B. Pandey and D. Stauffer, Physica A 289, 223 (2001)

[14] I. Chang and D. Stauffer, Physica A 264, 294 (1999)

[15] D. Stauffer and A. Aharony, Introduction to Percolation Theory, Taylor and Francis, London 1994; M. Sahimi, Applications of Percolation Theory, Taylor and Francis, London 1994; A. Bunde and S. Havlin, Fractals and Disordered Systems, Springer, Berlin-Heidelberg 1996

[16] Y.-C. Zhang, Physica A 269, 30 (1999)

[17] D. Stauffer and D. Sornette, Physica A 271, 496 (1999)

[18] D. Stauffer and N. Jan, Physica A 277, 215 (2000)

[19] B.M. Roehner, Int. J. Mod. Phys. C 11, 91 (2000) 
[20] P. Gopikrishnan, V. Plerou, X. Gabaix and H.E. Stanley, Phys. Rev. 62, R4493 (2000)

[21] N. Vandewalle and M. Ausloos, Eur. Phys. J. B 4, 257 (1998)

[22] F. Castiglione and D. Stauffer, Physica A, in press

[23] J.-P. Bouchaud, M. Potters and M. Meyer, Eur. Phys. J. B 13, 595 (2000)

[24] A. Johansen and D. Sornette, Eur. Phys. J. B 1, 143 (1998)

[25] K. Ide and D. Sornette, cond-mat/0106047; D. Sornette and K. Ide, condmat/0106054 